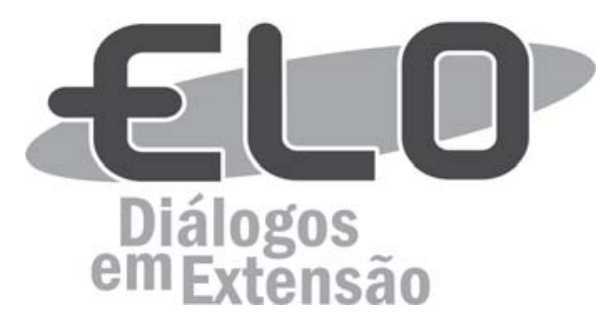

\title{
A prática das oficinas terapêuticas aplicadas ao Centro de Atenção Psicossocial no Vale do Jequitinhonha
}

\author{
Nadaby de Oliveira Matos ${ }^{1}$, Ana Luísa de Paulo Caldeira² e Antônio Moacir de Jesus Lima
}

\begin{abstract}
Resumo: Trata-se de um relato de experiência de acadêmicos do Curso de Graduação em Enfermagem como monitores das oficinas terapêuticas e no horizonte que se abre no contexto do tratamento psiquiátrico por meio da realização das mesmas. As oficinas ocorreram no Centro de Atenção Psicossocial Renascer, na região do vale do Jequitinhonha em Minas Gerais, e se caracterizaram como agentes potencializadores da reinserção social, buscando sempre estabelecer a autonomia dos usuários, estímulo do autocuidado e interação com o processo medicamentoso e psicoterápico do tratamento adotado.
\end{abstract}

Palavras-chave: Psicossocial. Oficinas. Reabilitação.

Área Temática: Saúde. Cultura.

\section{The practice of therapeutic workshops applied to the Center for Psychosocial Care in the Vale do Jequitinhonha}

\begin{abstract}
It is an experience report of students of undergraduate degree in nursing as monitors of terapeutics workshops and the oppening of horizon on context of psychiatric treatment by means of carrying them out. The workshops happened in Renascer psychosocial care Center, in the region of Vale do Jequitinhonha in Minas Gerais which were characterized as potentiating agents of social reintegration, always searching for establishing the autonomy of the users, for encouraging the self-care and for interacting with the medicative and psychotherapeutic process of the adopted treatment.
\end{abstract}

Keywords: Psychosocial. Workshops. Rehabilitation.

\section{La práctica de los talleres terapéuticos aplicados al Centro de Atención Psicosocial en el Vale do Jequitinhonha}

Resumen: Se trata de un relato de experiencia de académicos del Curso de Graduación de Enfermería como monitores de talleres terapéuticos y en el horizonte que se abre en el contexto del tratamiento psiquiátrico por medio de la realización de los mismas. Los talleres ocurrieron en el Centro de Atención Psicosocial Renascer, en la región del Vale do Jequitinhonha en Minas Gerais donde se caracterizaron como agentes potencializadores de la reinserción social, buscando siempre establecer la autonomía de los usuarios, estímulo del autocuidado e interacción con el proceso medicamentoso y psicoterápico del tratamiento adoptado.

Palabras clave: Psicosocial. Talleres. Rehabilitación.

\footnotetext{
${ }^{1}$ Bacharel em Enfermagem, Mestrando em Saúde, Sociedade e Ambiente pela Universidade Federal dos Vales do Jequitinhonha e Mucuri. Campus JK. Avenida Tijucana, 407, Bela Vista- Diamantina MG, CEP 39100-000. Contato: (38) 988463864 - [mattos_nadaby@hotmail.com]

${ }^{2}$ Discente do curso de Enfermagem, Universidade Federal dos Vales do Jequitinhonha e Mucuri. Campus JK.

${ }^{3}$ Docente na Universidade Federal dos Vales do Jequitinhonha e Mucuri pelo Departamento de Enfermagem. Campus JK. Coordenador do Projeto. Especialista em Saúde da Família e Saúde Coletiva com ênfase em Saúde Mental (UFMG). Mestre em Ensino e Saúde (UFVJM). Doutorando pela Faculdade de Medicina (UFMG).
} 


\section{Introdução}

A política de saúde mental vigente é resultado da grande mobilização de clientes/usuários, trabalhadores e familiares que reivindicaram melhorias nos métodos de tratamento mental, defendendo os direitos humanos, superação da violência asilar e proteção da cidadania dos portadores de transtornos mentais. Essa mobilização foi um marco histórico para a sociedade que se iniciou ao final da década de 1970 com o objetivo de conquistar outro patamar de serviços prestados aos portadores de transtornos mentais, a defesa dos direitos dos usuários, além de buscar a extinção dos manicômios no país.

Esse processo de mudança representa a luta social antimanicomial e um projeto coletivamente elaborado de alternância no modelo de atenção e de gestão do cuidado, a Reforma Psiquiátrica, apresentada como a junção de mudanças de hábitos sociais, conhecimentos, valores culturais e na rotina das instituições e serviços; é no coletivo comunitário que a evolução do processo da Reforma Psiquiátrica progride, notado por contrariedades, angústias, hostilidades e incitações (BRASIL, 2004).

A partir de 1980, experiências municipais começaram o processo de desinstitucionalização de manicômios, promovendo serviços de atenção psicossocial para realizar a reinserção de usuários em seus territórios existenciais, direcionado a ultrapassar o modelo assistencial centralizado no hospital psiquiátrico e na sua ideologia de repudiar os portadores de transtornos mentais da sociedade (FAGUNDES JUNIOR et al, 2016). Foram interditados hospitais psiquiátricos mediante a expansão dos serviços diferenciados de cuidado que não violassem os direitos das pessoas em tratamento (BRASIL, 2013). Após inúmeros embates ideológicos e, com a perspectiva da construção de um Sistema Único de Saúde equânime e efetivo, foi sancionada em 2001 a Lei Federal n 10.216 conhecida como 'Lei da Reforma Psiquiátrica', que propõe um redirecionamento da assistência em saúde mental (BRASIL, 2001).

Considerando as mudanças propostas, os Centros de Atenção Psicossocial (CAPS), regulamentados pela Portaria n $336 / \mathrm{GM}$ de 19 de fevereiro de 2002, surgiram no intuito de propor estratégias de serviços comunitários que atuam como dispositivos de organização da atenção em saúde mental com ênfase para a reabilitação psicossocial (LEÃO, 2008; BRASIL, 2004), substituindo os antigos manicômios e trazendo um novo método de saúde no qual os pacientes não se distanciam dos familiares, mas reafirmam seus laços intercalando o centro de atenção psicossocial e seu lar, que é o precursor na evolução positiva do tratamento. Nesse contexto, o CAPS foi reconhecido na IV Conferência Nacional de Saúde Mental Intersetorial como dispositivo de atenção substitutivo, ressaltando sua função estratégica de articulador da rede de serviços e a necessidade de potencializar parcerias intersetoriais (BRASIL, 2010).

Os CAPS se constituem como lugar de referência e tratamento para indivíduos com sofrimento psíquico, cuja gravidade e/ou reincidência demandam muitas vezes um cuidado intensivo. A assistência deve ser prestada por multiprofissionais com ação interdisciplinar ao qual fazem parte enfermeiros, médicos, psicólogos, terapeutas ocupacionais, músico terapeutas, assistentes sociais entre outros profissionais e/ou voluntários que estejam dispostos a trabalhar focando na melhoria da doença e reinserção social do cliente de maneira articulada e integrada, implicando apropriações mais aprofundadas, críticas e não totalizantes. (CONSELHO FEDERAL DE PSICOLOGIA, 2013).

O modelo assistencial em saúde mental instituído vem impondo a necessidade de aprofundar a organização e oferta dos serviços (prevenção, promoção, redução de danos, reabilitação, reinserção social) e de transformações no processo de trabalho que compõem esse cenário (ARANHA e SILVA et $a l$, 2005). Em relação a este último ponto, entende-se que reformular o objeto de trabalho (antes a doença, agora o sujeito) implica em repensar os meios de intervenção tendo como conduta a adoção de tecnologias que respondam ao projeto proposto, rompam com a organização médica do serviço e possibilitam momentos de intersecção de "sujeitos instituintes", ou seja, de interação entre os dois sujeitos (usuário-trabalhador) que instituem necessidades, saberes e representações de maneira democrática e respeitosa (MERHY EE, 1997).

As oficinas terapêuticas em saúde mental são atividades realizadas em grupo com a presença e orientação de um ou mais profissionais, monitores e/ou estagiários. São estratégias de cuidado, interação e socialização, além de funcionarem como elementos organizadores dos serviços de atenção diária de saúde mental, as oficinas têm sido compreendidas como ambientes de produção e manejo de subjetividade, de reconstrução de vínculos entre os sujeitos em sofrimento psíquico e seus grupos sociais, além de irem ao encontro dos discursos de quem cuida e de quem é cuidado (VALLADARES et al, 2003). 
Ao tocante do direito do trabalho no Brasil, não podemos desconsiderar que a reestruturação produtiva do capital vem propiciando lugar a formas mais desregulamentadas de trabalho, diminuído de grande forma o conjunto de trabalhadores estáveis que se estruturava por meio de empregos formais (ANTUNES, 2011). Os portadores de transtornos mentais acabam sendo duplamente excluídos do mercado de trabalho, em primeiro lugar, pelo estigma da doença e em segundo, pela própria falta de capacitação para o trabalho (NASCIMENTO, 2009; MARTINS, 2009).

A partir desse fardo de exclusão e classificação estigmatizada por muitos em relação aos portadores de transtornos mentais, que muitas vezes os classificam como "anormais", os próprios sujeitos direcionamse a apresentar crenças a respeito das suas identidades. Ao adotarem o medo, a vergonha e o sentimento de desvalorização, os portadores de transtornos mentais sentem-se incapazes de serem produtivos, tanto do ponto de vista físico, laboral e cultural, estando "a margem da sociedade" (GOFFMAN, 1980).

O presente trabalho visa constatar a atuação do profissional enfermeiro no âmbito das oficinas e no horizonte que se abre no contexto do tratamento psiquiátrico por meio da realização das mesmas, identificando seu potencial para a promoção da reinserção social dos sujeitos, aliado ao processo medicamentoso.

\section{Metodologia}

Neste relato procuramos descrever as experiências, e os resultados vividos durante e após a monitoria de oficinas terapêuticas por acadêmicos bolsistas e voluntários de Enfermagem da Universidade Federal dos Vales do Jequitinhonha e Mucuri (UFVJM) que ocorreram no interior do CAPS Renascer na cidade de Diamantina/MG, vale do Jequitinhonha, na sala de terapia coletiva, três vezes por semana no período de Fevereiro de 2015 até Março de 2017 através do projeto "Oficinas Terapêuticas: uma possibilidade de tratamento para a psiquiatria", vinculado a pró-reitora de extensão da UFVJM na modalidade PROCARTE, que além da extensão em si, tem como intuito o apoio à cultura e à arte, fundamentando-se no Plano Nacional de Cultura. O CAPS em questão é classificado como tipo II, devido ao atendimento prestado prioritariamente a pessoas em intenso sofrimento psíquico decorrente de transtornos mentais graves e persistentes, sendo referência para cerca de 20 municípios na região do Vale do Jequitinhonha, onde está alocado.

Os pacientes vão ao CAPS a procura de tratamento, fora de estágios de crise, com ou sem os familiares e/ou acompanhantes. Eles foram diariamente convidados a participar das oficinas, sendo a adesão espontânea. A quantidade de clientes por oficina obteve média de dez pessoas entre usuários do CAPS e familiares. Essas foram monitoradas por uma profissional em terapia ocupacional, uma psicóloga da própria instituição de saúde e em média dois alunos de graduação em Enfermagem por oficina. A supervisão das ações promovidas ocorria por parte do professor do Departamento de Enfermagem da UFVJM, especialista na área e coordenador do projeto.

Inicialmente as propostas das atividades eram apresentadas pelos monitores após discussão com o coordenador do projeto e expostas a equipe de saúde do CAPS para análise da aplicabilidade, visando a seleção na busca da mais produtiva, baseadas nos distúrbios apresentados pelos pacientes em cada encontro.

\section{Discussão}

A iniciativa para a idealização do projeto partiu de um diagnóstico situacional por meio da observação das práticas ofertadas no serviço de saúde. Notava-se um quadro de estagnação de alguns pacientes ao serem inseridos na instituição psiquiátrica devido ao fato de não se sentirem à vontade ou acolhidos, permanecendo grande parte do tempo ocioso pela ausência de atividades diversificadas e cientes do estigma de incapacidade, que leva ao desejo de abandono do tratamento farmacológico estabelecido, sendo esse risco de abandono o estímulo principal para o desenvolvimento da proposta por parte da equipe executora do projeto.

Objetivou-se implantar as oficinas terapêuticas como possibilidade de tratamento vinculado ao processo terapêutico de cada usuário, devido à necessidade de aprofundar a organização e oferta dos serviços, e torná-los mais efetivos e atrativos, visto que a evasão do tratamento tradicional medicamentoso pelos clientes era frequente (BRASIL, MS, 2004). Visou-se a realização de atividades produtivas, e o estímulo ao exercício coletivo da cidadania promovido pela arte e cultura que permeava as relações, devido as origens intermunicipais dos participantes, aliadas ao reconhecimento e ao respeito 
das diversidades existentes no grupo, configurando também com ambientes de intensa comunicação e integração (IBIAPINA et al., 2017).

As oficinas terapêuticas têm como desígnio possibilitar a discussão da diversidade do dia a dia, com a manifestação dos seguintes temas: relações familiares, de afeto amoroso, sexualidade, amizades, trabalho, entretenimento, cultura e bem-estar. A expressão desses temas possibilita intervenções de saúde, pois propicia a criação de vínculos, além da produção artística, do discurso dos sujeitos e acompanhamento da evolução dos casos, que gera a percepção dos sentidos e sentimentos conferidos pelos clientes ao seu tratamento e ao CAPS; e oportuniza a procura de significado existencial e de júbilo através das atividades em grupo, com a cultura e as artes (SOUZA, 2012).

O desenvolvimento desse método terapêutico permite a possibilidade de projeção de conflitos internos/externos por meio de atividades artísticas, com a valorização do potencial criativo, imaginativo e expressivo do usuário, além do fortalecimento da autoestima e da autoconfiança, a miscigenação de saberes e a expressão da subjetividade (AZEVEDO, 2010), não tendo como finalidade substituir as condutas dos serviços, mas integrá-las para melhor reabilitação do paciente. O desdobramento das abordagens terapêuticas no trabalho em saúde mental ocorre com intuito de melhorar o enfrentamento do transtorno psíquico (AMARANTE, 2007). NORONHA et. al. (2016) afirma em seu estudo com os familiares de pacientes adolescentes com transtornos mentais, que houve relatos positivos sobre a participação dos adolescentes nas oficinas terapêuticas, destacou que os clientes produziam manifestações afetivas, ajudavam no enfrentamento dos problemas do dia a dia, melhoravam a autoestima, o diálogo, a desinibição, a proatividade, acalmavam e, acima de tudo, auxiliavam na convivência familiar e na escola. Essa afirmação embasa os motivos pelos quais essa metodologia de tratamento tem resultados positivos no cuidado do paciente com transtornos mentais, sendo o cuidado a área de competência do profissional Enfermeiro. Os acadêmicos em Enfermagem da UFVJM participarem de um projeto de extensão com ênfase no cuidado, possibilitando um crescimento e amadurecimento considerável para o futuro profissional da saúde.

Foram empregadas no projeto a terapia psicossocial, que proporcionou diversas formas de atividades, práticas motoras (esportes, trabalhos em madeira, couro, papel, emborrachados), sociais (comemorações festivas, teatros, cinema, realização de eventos culturais) e expressivas (atividades espontâneas como cerâmica, pintura, dança, jogos e musicalização), além da reciclagem, que possui vantagens como: a minimização da utilização de fontes naturais, muitas vezes não renováveis e, da quantidade de resíduos que necessitam de tratamento final, como aterramento ou incineração, conferindo através de dobraduras, pintura, recorte e colagem a conscientização ecológica dos agentes envolvidos. Essas intervenções ampliam a habilidade e a autonomia do sujeito ao permitirem-no o desenvolvimento do potencial da criatividade e da expressão.

A arte é capaz de produzir subjetividades, catalisar afetos, engendrar territórios desconhecidos e/ ou inexplorados. O seu valor na reabilitação social está na possibilidade do usuário trabalhar e descobrir suas potencialidades para conquistar espaços sociais (TAVARES CMM, 2003). Optou-se pela simplicidade para gerar bem-estar e promover transformação social, conforme ilustrado nas figuras 1 e 2 .

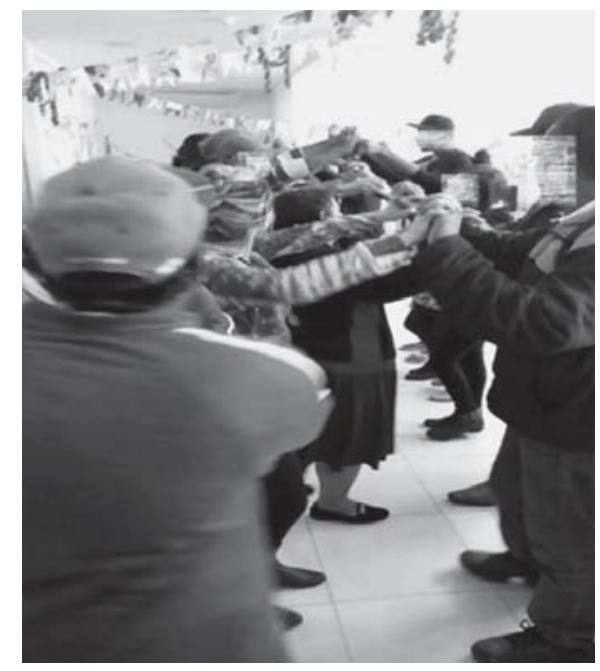

Figura 1 - Foto da Quadrilha junina realizada em 2016 com os participantes do projeto.

Fonte: Banco de dados do autor." 


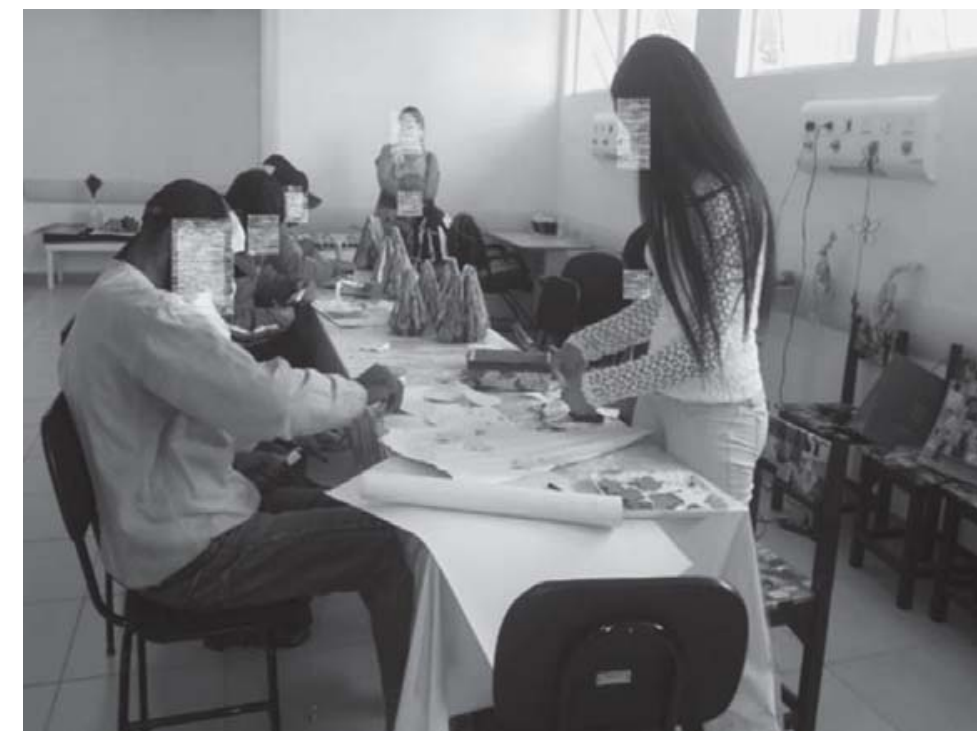

Figura 2 - Foto do processo de confecção Artístico em 2016.

Fonte: Banco de dados do autor.

\section{Resultados}

As exposições do material confeccionado ocorreu em eventos culturais, artísticos e científicos da própria Universidade e em pontos estratégicos e turísticos da cidade de Diamantina, MG, permitindo o reconhecimento da sociedade para com o CAPS, dos indivíduos envolvidos, do intercâmbio cultural em nível nacional e internacional e da terapêutica adotada, demonstrando assim que o doente mental possui contrariamente à crença popular, aptidões, competências e cultura, estando apto para exercer funções na sociedade, que evidenciam seu valor e reforçando a sua autoestima.

Obteve-se a desapropriação do campo conceitual de oficinas, muitas vezes associada somente ao conceito superficial da arte, saindo da forma incipiente do seu significado, contemplando o potencial e os reflexos positivos que a sua utilização como tratamento em Centros de Atenções Psicossociais é efetiva e crucial na complementação do tratamento e na terapêutica psicofarmacológica ofertada pelas instituições em saúde.

\section{Considerações finais}

Pelas singularidades existentes no CAPS percebe-se que o trabalho da Enfermagem se insere em uma prática que vai além dos recursos assistenciais tradicionais, desempenham ações em conjunto, desenvolvendo um trabalho com características coletivas e interdisciplinar na busca da reabilitação psicossocial.

A inclusão social por meio das oficinas terapêuticas exerce papel de suma importância no processo de eliminação do estigma social presente no âmbito da saúde mental e principalmente perante a sociedade. A cultura, sentimentos, anseios e emoções dos pacientes são bem representados nas práticas oficineiras, caracterizando como divisor de águas para um diagnóstico psíquico preciso, devido a transmissão de informações não perceptíveis em uma consulta tradicional.

A expressão de se sentir capaz e integrante de um meio social é nítido na feição e manifestações dos pacientes nos momentos de confecção e exposição a comunidade. É necessário que os avanços continuem a serem buscados e parcerias com a extensão universitária sejam mantidas a fim de que ações efetivas em saúde inovem e alcancem resultados ainda mais satisfatórios e contribuam também a formação dos acadêmicos envolvidos nas ações.

\section{Fonte de Financiamento}

Recursos financeiros e bolsa de extensão obtidos junto à Pró-reitora de Extensão (PROEXC) da Universidade Federal dos Vales do Jequitinhonha e Mucuri na modalidade PROCARTE. 


\section{Agradecimentos}

Agradecemos a todos os acadêmicos do curso de Enfermagem da UFVJM que se envolveram com as atividades nos estágios das disciplinas relacionadas a Saúde Mental ofertadas no curso. A próreitora de extensão e cultura (PROEXC) pelo incentivo e apoio, a Prefeitura Municipal de Diamantina/ MG e aos profissionais dos Centro de Atenção Psicossocial Renascer pela parceria e disposição no auxílio em todas as atividades.

\section{Referências}

AMARANTE P. Saúde Mental e Atenção Psicossocial. Rio de Janeiro: Editora Fiocruz; 2007.

ANTUNES, R. Os modos de ser da informalidade: rumo a nova era da precarização estrutural do trabalho? Serviço Social e Sociedade, São Paulo - SP. v1, n.107, p.-405-419. 2011.

ARANHA e SILVA, A.L; FONSECA, R.M.G.S. O processo de trabalho em saúde mental e o campo psicossocial. Revista Latino Americana de Enfermagem. v.13, n.3, p.441-9. 2005.

AZEVEDO, D.M; MIRANDA F.A.N. Práticas profissionais e tratamento ofertado nos CAPSad do município de Natal-RN: com a palavra a família. Escola Anna Nery. V.14, n.1, p.56-63. 2010.

BRASIL, Ministério da Saúde. Saúde Mental no SUS: os Centros de Atenção Psicossocial. Brasília-DF: Ministério da Saúde. p 20-26, 2004.

BRASIL. Ministério da Saúde. Portaria $n^{0}$ 224/MS, de 29 de janeiro de 1992. Brasília-DF: Ministério da Saúde, 1992. Disponível em: http://bvsms.saude.gov.br/bvs/publicacoes/ legislacao_saude_mental_1990_2004_5ed.pdf. Acesso em: 12 Fev. 2016.

BRASIL. Ministério da Saúde. Secretaria de Atenção à Saúde. Caderno de Atenção Básica. Departamento de Atenção Básica, 2013. Disponível em: http://bvsms.saude.gov.br/bvs/publicacoes/ cadernos_atencao_basica_34_saude_mental.pdf. Acesso em: 10 Abr. 2017.

BRASIL. Ministério da Saúde. Secretaria de Atenção à Saúde. DAPE. Coordenação Geral de Saúde Mental. Reforma psiquiátrica e política de saúde mental no Brasil. Documento apresentado à Conferência Regional de Reforma dos Serviços de Saúde Mental: 15 anos depois de Caracas. OPAS. Brasília, novembro de 2005. Disponível em: http://bvsms.saude.gov.br/bvs/publicacoes/ Relatorio15_anos_Caracas.pdf. Acesso em: 10 Maio 2018.

BRASIL. Ministério da Saúde; Conselho Nacional de Saúde. Relatório final da $4^{a}$ Conferência Nacional de Saúde Mental. Brasília-DF; 2010.

BRASIL. Ministério da Saúde; Secretaria de Atenção à Saúde; Departamento de Ações Programáticas Estratégicas. Saúde Mental no SUS: os Centros de Atenção Psicossocial. Brasília; 2004.

BRASIL. Presidência da República. Lei no 10.216, de 06 de abril de 2001. Brasília-DF: Presidência, 2001. Disponível em: http://bvsms.saude.gov.br/bvs/publicacoes/legislacao_saude_mental_ 1990_2004_5ed.pdf. Acesso em: 6 Abr. 2016.

CONSELHO FEDERAL DE PSICOLOGIA. Referências Técnicas para Atuação de Psicólogas(os) no CAPS Centro de Atenção Psicossocial, $1^{a}$ Edição. Brasília-DF: CFP, 2013. Disponível em: http:// www.crpsp.org.br/portal/comunicacao/diversos/cd-saude mental/publica\%C3\% A7\%C3\%B5es/ CREPOP/CAPS.pdf . Acesso em: 2 Abr. 2016.

FAGUNDES JUNIOR, Hugo Marques; DESVIAT, Manuel; SILVA, Paulo Roberto Fagundes da. Reforma Psiquiátrica no Rio de Janeiro: situação atual e perspectivas futuras. Ciênc. saúde coletiva, Rio de Janeiro, v. 21, n. 5, p. 1449-1460, maio 2016. Disponível em <http://www.scielo.br/ scielo.php?script=sci_arttext\&pid=S141381232016000501449\&lng=pt\&nrm=iso $>$. Acesso em: 10 Maio 2018.

GOFFMAN, E. Estigma: notas sobre a manipulação da identidade deteriorada. Rio de Janeiro: Zahar. 1980. 
IBIAPINA, A. R. S.; MONTEIRO, C. F. S.; ALENCAR, D. C. M.; FERNANDES, A.; COSTA FILHO, A. A. I. Oficinas Terapêuticas e as mudanças sociais em pacientes com transtorno mental. Escola Anna Nery. v. 21, n.3. p.1-7. 2017.

LEÃO, A; BARROS, S. As representações sociais dos profissionais de saúde mental acerca do modelo de atenção e as possibilidades de inclusão social. Saúde Sociedade. V. 17, n.1, p.95-106. 2008.

MARTINS, R. C. A. Cooperativas sociais no Brasil: debates e práticas na tecitura de um campo em construção. Dissertação (Mestrado em Ciências Sociais) - Instituto de Ciências Sociais, Universidade de Brasília, Brasília-DF. 2009.

MERHY, E. E. Em busca do tempo perdido: a micropolitica do trabalho vivo em saúde. In: Merhy EE, Onocko R, organizadores. Agir em saúde: um desafio para o público. São Paulo, Buenos Aires: Hucitec, Lugar Editorial; 1997.

NASCIMENTO, M. A. Erving Goffman, as interações no cotidiano escolar, desvendando o estigma dentro da inclusão escolar. Dissertação (Mestrado em Educação) - Faculdade de Humanidades e Direito da Universidade Metodista de São Paulo, São Bernardo do Campo/SP. 2009.

NORONHA, A A.; FOLLE, D.; GUIMARAES, A.N.; BRUM, M.L.B.; SCHNEIDER, J.F.; MOTTA, M.G.C. Percepções de familiares de adolescentes sobre oficinas terapêuticas em um centro de atenção psicossocial infantil. Revista Gaúcha de Enfermagem. V. 37; n.4; p.1-8. Porto Alegre 2016.

SOUZA, L. G. S., PINHEIRO L. B. Oficinas terapêuticas em um Centro de Atenção Psicossocial - álcool e drogas. Revista Aletheia [online]. v.38, n. 39, p.218-227. 2012..

TAVARES, C.M.M. O papel da arte nos centros de atenção psicossocial - CAPS. Revista Brasileira de Enfermagem. v.56, n.1, p. 35-9. 2003.

VALLADARES, A.C.A. et al. Reabilitação psicossocial através das oficinas terapêuticas e/ou cooperativas sociais. Revista Eletrônica de Enfermagem. v.5, n.1, p.4-9. 2003. 